\begin{tabular}{|c|l|}
\hline Title & Self-Ordering of Nanofacets on Vicinal Sic Surfaces \\
\hline Author(s) & Nakagawa, Hiroshi; Tanaka, Satoru; Suemune, Ikuo \\
\hline Citation & $\begin{array}{l}\text { Physical Review Letters, 91(22), 226107 } \\
\text { https://doi.org/L0.1103/PhysRevLett.91.226107 }\end{array}$ \\
\hline Issue Date & 2003-11-26 \\
\hline Doc URL & http://hdl.handle.net/2115/5646 \\
\hline Rights & Copyright $\odot 2003$ A merican Physical Society \\
\hline Type & article \\
\hline File Information & PRL91-22.pdf \\
\hline
\end{tabular}

Instructions for use 


\title{
Self-Ordering of Nanofacets on Vicinal SiC Surfaces
}

\author{
Hiroshi Nakagawa, Satoru Tanaka, ${ }^{*}$ and Ikuo Suemune \\ Research Institute for Electronic Science, Hokkaido University, Sapporo 060-0812, Japan
}

(Received 22 May 2003; published 26 November 2003)

\begin{abstract}
Vicinal $4 H$ and $6 H$-SiC(0001) surfaces have been investigated using atomic force microscopy and cross-sectional high-resolution transmission electron microscopy. We observed the characteristic selfordering of nanofacets on any surface, regardless of polytypes and vicinal angles, after gas etching at high temperature. Two facet planes are typically revealed: (0001) and high index $(11 \overline{2} n)$ that are induced by equilibrium surface phase separation. A $(11 \overline{2} n)$ plane may have a free energy minimum due to attractive step-step interactions. The differing ordering distances in $4 H$ and $6 H$ polytypes imply the existence of $\mathrm{SiC}$ polytypic dependence on nanofaceting. Thus, it should be possible to control $\mathrm{SiC}$ surface nanostructures by selecting a polytype, a vicinal angle, and an etching temperature.
\end{abstract}

DOI: $10.1103 /$ PhysRevLett.91.226107

Self-ordering or self-organization on semiconductor surfaces is attracting a lot of attention because of its potential use in patterning and fabrication of nanometer scale semiconductor device structures [1] as a replacement for conventional lithographic techniques in next generation integrated circuit manufacture. Periodically and spontaneously ordered semiconductor surfaces reveal significantly fine and spatially uniform patterns, which are far beyond the lowest limit of electron beam induced patterning technique. Two-dimensional ordering is the ultimate goal in the future applications [2,3]. However, one-dimensional ordering of semiconductor surfaces on a nanometer scale is still of benefit for drastically improving device performance and may, in addition, reveal new physical phenomena. It is known that semiconductor surfaces show ordering phenomena such as surface reconstruction, step/terrace formation, faceting, domain formation, etc. [1]. Formation of step/terrace structures is commonly observed on vicinal surfaces [4]. Technologically important substrates such as $\mathrm{Si}$ [5-7] and GaAs [8-10] have been studied, both for device applications and for the understanding of the basic surface physics such as step energetics and surface selfordering mechanisms. The heteroepitaxial growth of metals and semiconducting materials on such ordered surfaces has also been investigated in order to achieve ordered nanostructures [1].

Silicon carbide is an interesting compound semiconductor, which possesses unique physical characteristics such as polymorphism, wide band gap, high melting point, and significantly superior electronic properties [11]. It is thus a promising semiconductor for the next generation of high power and high frequency electronic devices [12] and also for a strong candidate as the substrate for III-nitride epitaxy [13]. Substrates with polytypes of $4 H$ and $6 H$-SiC are commercially available and now widely used for these purposes. Chemically and structurally abrupt and defect-free interfaces are required in such devices, making the surface of the $\mathrm{SiC}$ substrate of great importance. There have been many reports con-
PACS numbers: 68.37.Ps, 68.35.Md, 68.37.Lp, 68.65.-k

cerning surface cleaning and smoothing [14-19], potentially as a consequence of the rather rough and scratched features exhibited by commercially available $\mathrm{SiC}$ substrates [15]. High temperature gas etching using $\mathrm{H}_{2}$ [16-18], $\mathrm{H}_{2} / \mathrm{HCl}[15,19]$, and $\mathrm{H}_{2} / \mathrm{C}_{2} \mathrm{H}_{4}[16,19]$ has been shown to be effective in attaining atomically smooth surfaces. Bunched steps and terraces were typically observed on on-axis $\mathrm{SiC}(0001)$ surfaces after such treatments. Step bunching is commonly observed and has been explained in part in terms of etching kinetics and surface energies by Nakamura et al. [15]. Interestingly, the steps are regularly spaced and their edges are fairly straight. Ohtani et al. suggested that the ordering of steps on on-axis $\mathrm{SiC}$ surfaces relies on short-range order stepstep interactions [20]. However, very few papers have reported on vicinal (off-axis) SiC surfaces, concerning surface evolution after etching, probably due to insufficient structural quality and an increased difficulty in obtaining smooth features at the relatively high etching temperatures $>1500{ }^{\circ} \mathrm{C}$, compared to temperatures required for on-axis surfaces.

In this Letter, we demonstrate and discuss periodically faceted surface morphologies on both $4 H_{-}$and $6 H$-SiC(0001) with several vicinal angles. Detailed structural studies are presented, using atomic force microscopy (AFM) and high-resolution transmission electron microscopy (HRTEM).

Samples used in this study are commercially available $\mathrm{Si}$ face of $4 H$ - and $6 H$-SiC(0001) substrates. Four samples have been investigated, including $4 H-\mathrm{SiC}$ (on and $8^{\circ}$ off) and $6 \mathrm{H}$-SiC (on and $3.5^{\circ}$ off). The off-axis substrates are all tilted toward [1120]. All as-received substrates show scratched features, which normally remain after standard polishing processes [15]. After the chemical cleaning procedures, comprising trichroloethylene, acetone, and methanol in an ultrasonic bath followed by $50 \% \mathrm{HF}$ etching to remove surface oxides, the substrate is loaded into a horizontal cold-wall reactor whose temperature is monitored by a thermocouple at the SiC-coated graphite susceptor. The $\mathrm{HCl}(0 \%-0.1 \%) / \mathrm{H}_{2}$ gas etching is then 

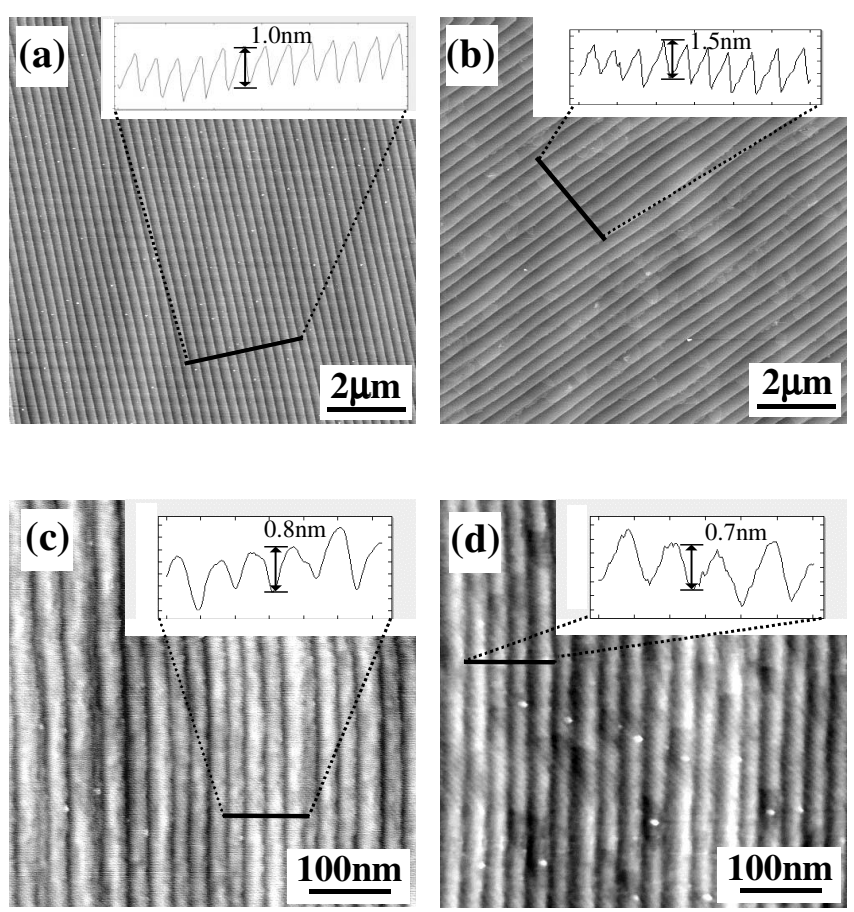

FIG. 1. AFM images of (a) on-axis $4 H$-SiC, (b) on-axis $6 H$-SiC, (c) $8^{\circ}$ off $4 H-\mathrm{SiC}$, and (d) $3.5^{\circ}$ off $6 \mathrm{H}$-SiC surfaces after $\mathrm{H}_{2} / \mathrm{HCl}$ gas etching. Inset figures show the results of the cross-sectional height analysis across the line indicated in each figure.

performed at $1430{ }^{\circ} \mathrm{C}$ at 760 Torr for $15-30 \mathrm{~min}$. Finally, the samples are examined by AFM (Nanoscope III) and HRTEM (Topcon EM-002B).

Figures 1(a)-1(d) show typical AFM images of SiC surfaces after etching. Surface scratches are removed and clear step/terrace structures are observed on all substrates. It is noted that the steps in each surface are very straight along [1100] within the AFM resolution and are fairly equally spaced, implying one-dimensional ordering of steps. In the case of on-axis substrates shown in Figs. 1(a) and 1(b), the step height is 1.0 and $1.5 \mathrm{~nm}$ in $4 H-\mathrm{SiC}$ and $6 H-\mathrm{SiC}$, respectively (see the figure insets), corresponding to the height of one-unit cell in each polytype. This is due to step bunching, which has been reported by several authors $[15,18,20]$.

Vicinal surfaces, on the other hand, show very narrow terraces, whose width is $\sim 28 \mathrm{~nm}$ in both polytypes, and bunched steps, as indicated in Figs. 1(c) and 1(d). Note that the terrace width is about the same in each sample regardless of the vicinal angles $\left(8^{\circ}\right.$ and $\left.3.5^{\circ}\right)$ and the height of bunched steps $(h)$ is measured to be less than one-unit cell height, unlike the on-axis surfaces (see the figure insets). The estimated terrace widths, assuming the simple step geometry having one-unit cell height of steep cliffs [i.e., perpendicular to (0001)], are 7.2 and $24.6 \mathrm{~nm}$ in $8^{\circ}$ off $4 \mathrm{H}-\mathrm{SiC}$ and $3.5^{\circ}$ off $6 \mathrm{H}-\mathrm{SiC}$, respectively. This implies that these vicinal surfaces should not have such a simple step geometry but a more complex structure as

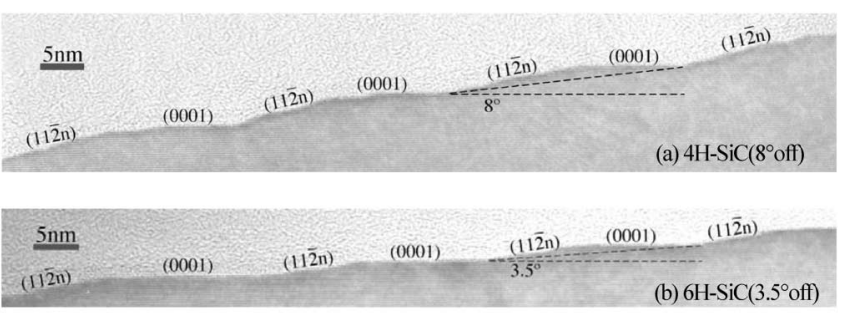

FIG. 2. Cross-sectional HRTEM images of (a) $8^{\circ}$ off $4 H$-SiC and (b) $3.5^{\circ}$ off $6 \mathrm{H}$-SiC surfaces. The ordering of facets in nanometer scale (nanofaceting) is clearly observed. Note that the vicinal angle $\left(3.5^{\circ}\right.$ or $\left.8^{\circ}\right)$ is conserved.

will be shown in cross-sectional HRTEM images in the following section.

Figures 2(a) and 2(b) show cross-sectional HRTEM images of $4 H-\mathrm{SiC}$ ( $8^{\circ}$ off $)$ and $6 H-\mathrm{SiC}\left(3.5^{\circ}\right.$ off $)$, respectively, which are viewed from the direction perpendicular to the tilting direction of [1120]. The periodical faceting in nanometer scale (nanofaceting) is clearly observed. Basal (0001) planes and high-index $(11 \overline{2} n)_{4 H}$ or $6 H$ facets appeared alternately with fairly regular periodicity, where $n$ is defined by the angle between a basal plane and a facet. The averaged periodicity is measured by AFM to be $\sim 28 \mathrm{~nm}$ in both substrates, close to the terrace width. Thus, we can confirm that the terrace width measured by AFM corresponds to the faceting periodicity observed by TEM. The typical angles of $(11 \overline{2} n)$ facets are $15^{\circ}-16^{\circ}$ and $13^{\circ}-14^{\circ}$ in $4 H-$ and $6 H-S i C$ which correspond to $n=11-12$ and $n=16-21$, respectively [21]. Note that the vicinal angle is always conserved and the areas of nanofacets exposed are determined by this conservation [22]. Moreover, presence of a highindex $(11 \overline{2} n)_{4 H \text { or } 6 H}$ facet is also confirmed on the onaxis $\mathrm{SiC}$ surfaces.

The detailed structure of a facet is investigated by HRTEM using a zone axis of [1 $1 \overline{100}]$, in which (0006) or (0004) lattice fringes can be imaged. In Fig. 3, a typical lattice image in $3.5^{\circ}$ off the $6 \mathrm{H}-\mathrm{SiC}$ surface region is shown. A facet consists of several bimonolayer steps and (0001) terraces of $\sim 1 \mathrm{~nm}$ width. This surface configuration is promoted energetically by step-step interactions, resulting in the $(11 \overline{2} n)$ facet formation.

Based on the analyses performed above the nanofacet structure in terms of the step height $(h)$ measured by AFM, the periodicity ( $w)$, and a "real" bunched step

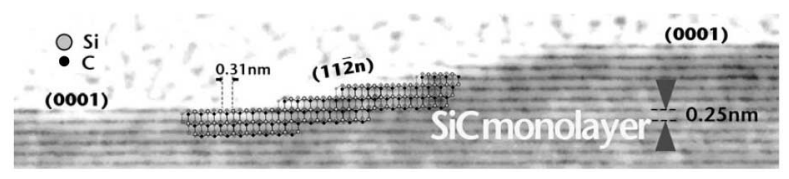

FIG. 3. A cross-sectional HRTEM image of (112 $n$ ) facet observed in a $3.5^{\circ}$ off $6 \mathrm{H}$-SiC surface. The atomic drawing of the lattice is superimposed. 


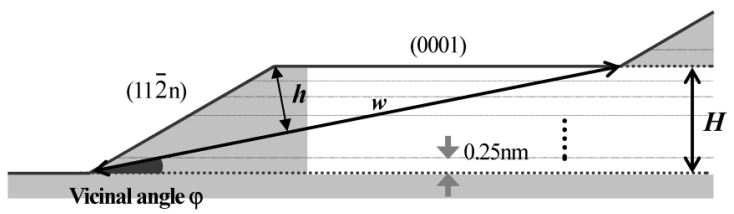

FIG. 4. Schematic illustration of a nanofacet, based upon the analysis of HRTEM. $\varphi$ is the vicinal angle of the substrate, $H$ is the "real" step height resulting from bunching (or faceting), $w$ is the periodicity of nanofaceting, and $h$ is the height of nanofacets measured by AFM.

height $(H)$ is schematically shown in Fig. 4. The statistical analysis of $w$ to examine the distribution of $H$ was then performed by AFM over a wide surface area more than $5 \mu \mathrm{m}^{2}$. The results are shown in Fig. 5(a) and 5(b). The insets show a typical feature of a pair of nanofacets in each substrate. The peak position of the distribution of $w$ is approximately the same in each sample even if the vicinal angle is different. This indicates that the step bunching is more pronounced in the $8^{\circ}$ off $4 H$-SiC surface than in the $3.5^{\circ}$ off $6 \mathrm{H}-\mathrm{SiC}$ surface as indicated by the height, $N$, of the bunched step; $N=4$ for $4 H-S i C$ and $N=1$ for $6 H$-SiC, where $N$ is in a unit of one-unit cell of each polytype. Note that the stable surface after bunching is always $A B A^{\prime} C^{\prime}$ in $4 H$ and $A$ of $A B C A^{\prime} C^{\prime} B^{\prime}$ in $6 H$, where $A B C$ denotes the classical notation of the stacking sequence of $\mathrm{SiC}$ polytypes [23]. This is possibly a result of surface energy variation in each plane as predicted by Cheng et al. [24] and Chien et al. [25]. Moreover, an obvious difference was noted in the degree of fluctuation of $w$ between the two samples. These results could suggest polytypic and vicinal angle dependences of nanofaceting. However, it is difficult to draw any conclusion on these topics at present without further experimentation.

Now we consider the self-ordering mechanism of nanofaceting observed on vicinal $\mathrm{SiC}$ surfaces in terms of surface free energy [22] and surface elasticity theory $[1,26]$. The former gives energetic stability of a $(11 \overline{2} n)$ facet and a (0001) plane: equilibrium surface phase separation. The latter suggests the origin of the ordering of nanofacets. Figure 6 shows a free energy curve schematically as a function of the vicinal angle $\varphi$ between a basal (0001) plane and a facet. The surface free energy is lowest at (0001) and monotonically increases as the vicinal angle $\varphi$ is increased as shown by the dashed curve [22]. We then introduce the concept of an energy minimum at the vicinal angle of $13^{\circ}-14^{\circ}$ (in the case of $6 \mathrm{H}$-SiC), corresponding to a $(11 \overline{2} n)$ facet, predicted by the HRTEM and AFM observations. The surface energy reduction can be induced by the presence of attractive step-step interactions between closely spaced bimonolayer steps as shown in Fig. 3. This was also realized in faceting of $\mathrm{Si}(111)$ by Williams et al. [22]. As a result, the double concave feature of the surface free energy curve is obtained (see Fig. 6) and provides a plausible explanation of the surface

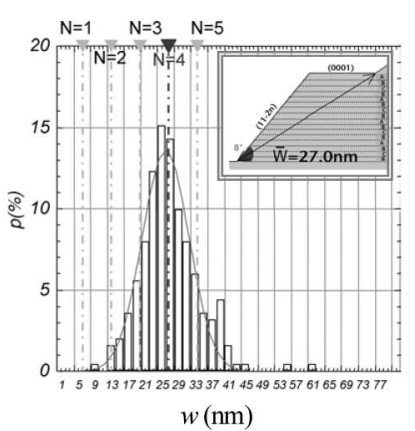

(a) $4 \mathrm{H}-\mathrm{SiC}\left(8^{\circ}\right.$ off $)$

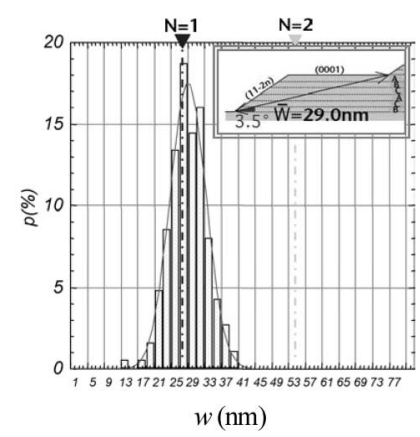

(b) $6 \mathrm{H}-\mathrm{SiC}\left(3.5^{\circ} \mathrm{off}\right)$
FIG. 5. Statistical analysis of the distribution of terrace width (w) on (a) $8^{\circ}$ off $4 H-\mathrm{SiC}$ and (b) $3.5^{\circ}$ off $6 H$-SiC surfaces. Schematic illustrations inserted show the typical facet geometries of off-axis $4 \mathrm{H}$-SiC and $6 \mathrm{H}$-SiC in which steps are energetically favored to bunch with one-unit cell height and four-unit cell height, respectively.

phase separation into two stable planes. Since the surface free energies of $3.5^{\circ}$ and $8^{\circ}$ off $\mathrm{SiC}$ surfaces are relatively higher than those of (0001) before relaxation, the surfaces will be separated into (0001) and $(11 \overline{2} n)$ facets as a result of the minimization of the surface free energy. The area ratio of $(0001)$ and $(11 \overline{2} n)$ facets is given by the lever rule.

The above discussion merely gives the area ratio of (0001) and $(11 \overline{2} n)$ facets but provides no mechanism for the ordering of nanofacets. This can be understood qualitatively from theoretical studies that have been developed for step-bunching phenomena. Essentially, the elastic dipole-dipole interaction at the edges of two different facets determines the ordering periodicity [26]. Shuchukin and Bimberg discussed periodic arrays of macroscopic step bunches based on the surface free energy described by the following equation [1]:

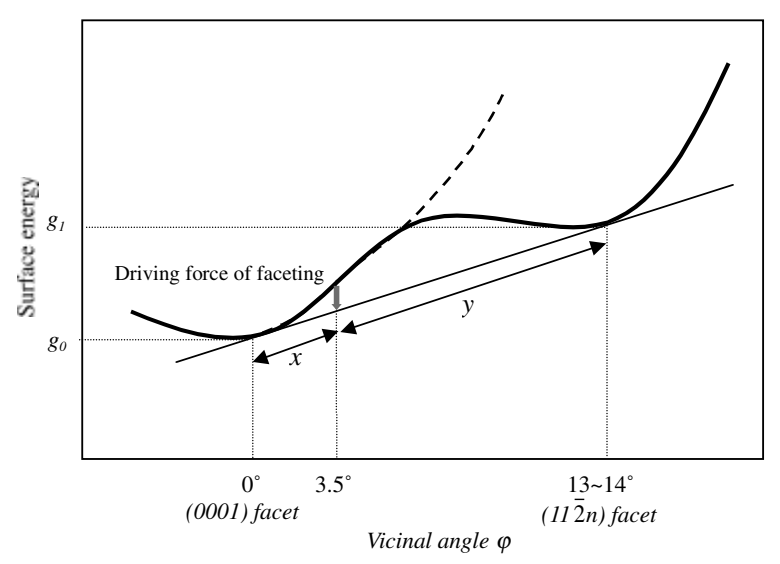

FIG. 6. Surface free energy curves as a function of vicinal angle $\varphi$. Dashed and solid curves indicate the surface free energy in the absence and in the presence of step-step interactions, respectively. The $x: y$ ratio represents the area ratio of $(0001)$ and $(11 \overline{2} n)$ facets. 


$$
E_{\text {total }}=E_{\text {terrace }}+E_{\text {facet }}+E_{\text {edges }}+\Delta E_{\text {elastic }}
$$

The surface free energy, $E_{\text {total }}$ is separated into $E_{\text {terrace }}$, $E_{\text {facet }}, E_{\text {edges }}$, and $\Delta E_{\text {elastic }}$, where $E_{\text {terrace }}, E_{\text {facet }}$, and $E_{\text {edges }}$ are the free energy of the terraces, facets, and edges, respectively, and $\Delta E_{\text {elastic }}$ is the elastic energy due to the discontinuity of the surface stress tensor at the step edges. $E_{\text {total }}$ can be written in the following equation [1]:

$$
E_{\text {total }}=\gamma_{0}+\gamma_{1}+\frac{C_{1} \eta}{D}-\frac{C_{2} \tau^{2}}{Y D} \ln \left(\frac{D}{a}\right) .
$$

Here $\tau$ is the characteristic value of the intrinsic surface stress tensor, $\gamma_{0}$ is the surface energy of a flat terrace, $\gamma_{1}$ is the surface energy of a step bunch, $\eta$ is the energy of two edges, $a$ is the lattice parameter, $Y$ is Young's modulus, $C_{1}$ and $C_{2}$ are geometric factors, and $D$ is the period of the faceted surface.

The minimized $E_{\text {total }}$ suggests the step configuration and then the optimum period of faceting, $D_{\text {opt }}$. The optimum period of nanofaceting is measured to be $\sim 28 \mathrm{~nm}$ on both $4 \mathrm{H}$ - and $6 \mathrm{H}$-SiC surfaces with the difference in step-bunching height $H$ as shown in Fig. 5. The question here is why the step bunching takes place in $4 \mathrm{H}-\mathrm{SiC}$ with the average height of bunched steps having a four-unit cell $(N=4)$. It is premature to discuss the physical reasons for this configuration; however, we may speculate that the bunched step configurations with an integer number $(N=1,2,3,4, \ldots)$ of unit cell height are energetically stabilized and each possess a slight difference in the total energy. At the annealing temperature used in this experiment, the stable surface geometry with $N=4$ is achieved, probably because the total energy is reduced as the bunched magnitude of $N$ increases. The slight energy difference between each configuration can result in the fluctuation of the periodicity as shown in Fig. 5(b). A similar scenario can be applied to $6 \mathrm{H}$-SiC but with a larger difference in the total energy in each bunched step configuration with $N=1,2,3, \ldots$ than in $4 H-\mathrm{SiC}$, suggesting the presence of a larger energy barrier transition to the surface configuration of $N=2$. This may originate from a polytypic dependence in SiC. Currently, the details of these topics are under investigation using $4 \mathrm{H}$ and $6 H$-SiC substrates with several vicinal angles $(\varphi=$ $\left.3.5^{\circ}, 8^{\circ}, 13^{\circ}\right)$.

In summary, vicinal $4 H$ - and $6 H-\mathrm{SiC}(0001)$ surfaces tilting toward $[11 \overline{2} 0]$ after high temperature gas etching are examined by means of AFM and HRTEM. All the surfaces investigated show ordered nanofacets, consisting of two planes; (0001) and high-index $(11 \overline{2} n)(n=11-12$ for $4 H$ and $n=16-21$ for $6 H$ ). This is induced by equilibrium surface phase separation. A $(11 \overline{2} n)$ plane, which has equally spaced $(\sim 1 \mathrm{~nm})$ steps with single bimonolayer height and (0001) terraces, may have a free energy minimum due to attractive step-step interactions. The self-ordering of nanofacets is discussed on the basis of the surface free energies including the elastic relaxation energy originating from the dipole-dipole interaction acting on the facet edges. The ordering periodicity is found to be dependent on the polytype $(4 H$ or $6 H$ ) as a result of the energetically stable facet configuration, determined by the height of bunched steps. The results obtained in this study could open up the way to control surface nanostructures in two dimensions by selecting a $\mathrm{SiC}$ polytype, a vicinal angle, and an etching temperature.

This work was supported in part by Grant-in-Aid (A) No. 14205001 from Ministry of Education, Culture, Sports, Science, and Technology of Japan.

*Author to whom correspondence should be addressed. Electronic address: stanaka@es.hokudai.ac.jp

[1] A. Shuchukin and D. Bimberg, Rev. Mod. Phys. 4, 71 (1999).

[2] H. Brune et al., Nature (London) 394, 451 (1998).

[3] D. Martrou, J. Eymery, and N. Magnea, Phys. Rev. Lett. 83, 2366 (1999).

[4] H.-C. Jeong and E. D. Williams, Surf. Sci. Rep. 34, 171 (1999).

[5] O. L. Alerhand et al., Phys. Rev. Lett. 64, 2406 (1990).

[6] H. Hibino et al., Phys. Rev. B 47, 13027 (1993).

[7] F. K. Men et al., Phys. Rev. Lett. 88, 096105 (2002).

[8] M. Kasu and N. Kobayashi, Appl. Phys. Lett. 62, 1262 (1993).

[9] M. D. Pashley and K.W. Haberern, Phys. Rev. Lett. 67, 2697 (1991).

[10] H. Hata et al., Phys. Rev. B 57, 4500 (1998).

[11] See, e.g., M. A. Capano and R. J. Trew, Mater. Res. Soc. Bull. 22, 19 (1997).

[12] Silicon Carbide and Related Materials 2001, edited by S. Yoshida, S. Nishino, H. Harima, and T. Kimoto (Trans. Tech. Publications, Zurich, 2002), Pt. 2.

[13] T.W. Weeks et al., Appl. Phys. Lett. 67, 401 (1995).

[14] R. Yakimova et al., Diam. Relat. Mater. 6, 1456 (1997).

[15] S. Nakamura et al., Appl. Phys. Lett. 76, 3412 (2000).

[16] A. Kawasuso et al., Appl. Phys. Lett. 76, 1119 (2000).

[17] F. Dulot et al., Appl. Surf. Sci. 187, 319 (2002).

[18] F. Owman et al., J. Cryst. Growth 167, 391 (1996).

[19] Z. Y. Xie et al., J. Cryst. Growth 217, 115 (2000).

[20] N. Ohtani et al., Phys. Rev. B 59, 4592 (1998).

[21] The measurement of the facet angle includes $\sim \pm 1 \%$ errors.

[22] E. D. Williams et al., Surf. Sci. 294, 219 (1993).

[23] N.W. Jepps and T. F. Page, in Progress in Crystal Growth and Characterization, edited by P. Krishna (Pergamon, New York, 1983) Vol. 7, p. 259.

[24] C. Cheng, R. J. Needs, and V. Heine, J. Phys. C 21, 1049 (1988).

[25] F. R. Chien et al., J. Mater. Res. 9, 4 (1994).

[26] V. I. Marchenko and A. Y. Parshin, Sov. Phys. JETP 52, 1 (1980). 